\title{
Neurocritical Care Resource Utilization in Pandemics: A Statement by the Neurocritical Care Society
}

\author{
Asma M. Moheet ${ }^{1 *}$ D, Angela H. Shapshak², Megan A. Brissie ${ }^{3}$, Yasser B. Abulhasann ${ }^{4}$ Gretchen M. Brophy $^{5}$, \\ Jennifer Frontera ${ }^{6}$, Wiley R. Hall ${ }^{7}$, Sayona John ${ }^{8}$, Atul A. Kalanuria ${ }^{9}$, Abhay Kumar ${ }^{10}$, Abhijit V. Lele ${ }^{11}$, \\ Shraddha Mainali ${ }^{12}$, Casey C. May ${ }^{13}$, Stephan A. Mayer ${ }^{14}$, Victoria McCredie ${ }^{15}$, Gisele S. Silva ${ }^{16}$, Jeffrey M. Singh ${ }^{15}$, \\ Alexis Steinberg ${ }^{17}$, Gene Sung ${ }^{18}$, Eljim P. Tesoro ${ }^{19}$ and Aleksandra Yakhkind ${ }^{20}$
}

๑ 2020 Springer Science+Business Media, LLC, part of Springer Nature and Neurocritical Care Society

\section{Background}

Global healthcare delivery has been impacted significantly by the millions of cases of COVID-19, the disease caused by SARS-CoV-2 [1]. Data from China suggest a potential association between COVID-19 mortality and health resource availability [2]. Projection models of hospital utilization during the COVID-19 outbreak have led to efforts to optimize critical care response and increase critical care resources [3]. In spite of robust and innovative attempts to increase the number of available intensive care unit (ICU) beds, appropriate allocation of medical resources is under constant flux, from rationing of personal protective equipment (PPE) to allocating ICU beds, ventilators, and staff to those who may benefit most [4]. Though cases of COVID-19 are currently increasing, individuals continue to suffer from other medical emergencies, including neurologic emergencies such as acute ischemic and hemorrhagic stroke, traumatic brain injury, traumatic spinal cord injury, and aneurysmal subarachnoid hemorrhage. Given the expected differences in resource availability and pandemic response across various institutions globally, the Neurocritical Care Society (NCS) has decided to put forth this document to provide key considerations for care of neurocritically ill patients during a pandemic.

\footnotetext{
${ }^{*}$ Correspondence: Asma.Moheet@OhioHealth.com ${ }^{1}$ Neurocritical Care, OhioHealth Riverside Methodist Hospital, 3535 Olentangy River Road, Columbus, OH 43214, USA Full list of author information is available at the end of the article
}

\section{Pre-hospital Systems of Care, Transfers, and Triage Practices Impacting Neurocritical Care}

Neurocritical care patients have improved outcomes when cared for by experienced neurocritical care providers. Admission to a neurocritical care unit (NCCU) rather than a non-specialty ICU reduces mortality for patients with intracerebral hemorrhage (ICH) [5]. The addition of a neurointensivist to a neurologic specialty ICU improves care quality, reduces cost, and enhances outcome [6]. There is also benefit in deploying a neurocritical care service in an institution previously without a subspecialty-led neurocritical care team for the care of patients with $\mathrm{ICH}$, ischemic stroke, traumatic brain injury (TBI), and subarachnoid hemorrhage (SAH) [715]. The most recent data in this area have demonstrated benefit in the USA and globally [16-23]. A framework for establishing various levels of neurocritical care based on regional resource availability has been previously proposed, with the goal of optimizing neurocritical care delivery through a team-based approach [24, 25].

Despite the stress on resources during a pandemic, pre-hospital efforts should focus on continued appropriate triage of neurocritically ill patients. Beyond routinely transferring patients to a higher level of care, alternate strategies may include retaining the patient at the presenting hospital and utilizing innovative methods to provide neurocritical care expertise through telemedicine. Regional surge planning should include coordination between government health authorities, neurocritical care leaders and hospital leadership to establish a graded escalation of response appropriate for varying surge 
levels [26]. Clinical pathways to determine pre-admission screening and/or testing for contagious illness should be clearly outlined for the neurocritical care patient who may require time-sensitive treatments. Similarly, patients with contagious illness should be monitored for development of neurologic symptoms. In addition, the need for geographic cohorting of patients awaiting test results or those later identified as positive, should be addressed. Finally, efforts should be made to educate the public that hospitals remain capable of caring for patients with neurologic emergencies safely with measures in place to protect patients against infection transmission, as there may be an increase in patients with concomitant neurologic emergencies such as stroke due to pandemic illness or conditions, and these patients may be hesitant to seek necessary emergency care due to fears of contracting an infectious illness [27].

\section{Inpatient Care of the Neurocritically-III Patient}

In a pandemic, the physical location of an NCCU may change based on an overall need to maintain certain resources for the care of infected patients, such as negative pressure isolation rooms. Given the evidence that neurocritical care expertise improves outcomes in those with neurologic emergencies, attempts should be made to cohort patients requiring admission to an NCCU to optimize care delivery by an experienced team and minimize the risks of care by those without neurocritical care expertise. In-hospital travel (changing room assignments, reducing off floor tests, etc.) should be minimized to decrease handoffs and decrease the risk of exposure to an infectious agent. To ensure availability of NCCU beds, PPE, and to minimize the risk of broad infectious exposure to and from patients, discussions with neurosurgical and neurointerventional colleagues should be held to outline criteria for potentially postponing nonemergent surgeries. Policies should be developed for the management of neurological emergencies (acute stroke responses, invasive procedures, and neurosurgical procedures) to ensure patient care needs are met, PPE is available, and team member exposure is limited.

It is important to consider the impact of a resource crisis on the patient experience. In pandemics, hospitals may limit or prohibit patient visitation. This necessary intervention unfortunately isolates patients from their community, and hampers communication between providers, patients and the patient's family or representatives. Attempts to reduce patient and clinician interactions may contribute to the patient's sense of isolation, and may unintentionally remove the humanistic interaction between a patient and their provider. Though direct patient examination by the provider is ideal, it may not always be feasible, and examinations via telemedicine with the assistance of a bedside provider may be required. Under these circumstances, providers should prioritize daily communication with the patient and the patient's next of kin or designated surrogate. For patients who are conscious, every effort should be made to facilitate phone or video interaction with their loved ones. Even in patients with altered sensorium or coma, the opportunity for family and/or surrogate decision makers to participate in audio-visual communication with the patient can carry substantial benefit.

Finally, prognostication early in the course of neurologic injury is challenging. Conversations surrounding goals of care should be shared between the neurocritical care team and the patient, family or surrogate decision makers while taking into account the best available medical evidence in the context of the patient's goals, values, and preferences [28]. To this end, it is vital to ensure a process for frequent communication with the patient's surrogate decision maker, and strategies such as telephone calls or videoconferencing (between members of the care team and these individuals or the patient and these individuals) should be considered. Disposition planning, such as discharge to rehabilitation hospitals, should continue to focus on facilities best-suited to maximize chances of long-term recovery.

\section{Neurocritical Care Physician and Advanced Practice Provider Staffing}

Resource crises place unprecedented demands on the healthcare workforce, forcing many facilities to reconsider staffing and scheduling models. Based on 2015 data from the American Hospital Association, there are approximately 28,808 active intensivists in the USA [29]. In addition, there are an estimated 34,000 critical care advanced practice providers (APPs) [30]. The number of critical care beds per capita can vary substantially from country to country, as well as the ability to staff these beds. The influx of patients requiring critical care services can overwhelm providers in disease epicenters. This shortfall is likely to be exacerbated in a pandemic as healthcare providers themselves fall ill. Consequently, it is necessary to consider alternate models of care to meet patient needs, optimize patient safety, and preserve clinician well-being. The Society for Critical Care Medicine (SCCM) has proposed a tiered staffing model, in which trained intensivists supervise teams comprised of critical care APPs, non-intensivist physicians, pharmacists, respiratory therapists, and nurses [31]. In this model, providers from a variety of backgrounds function as "intensivist-extenders" in order to provide critical care expertise to a much greater number of patients than would typically be feasible. In the event of such reorganization, a neurointensivist, as a critical care 
trained physician, may be needed to provide care for ICU patients without primary neurocritical illness. Neurocritical care physicians and APPs have robust foundations in general critical care, including the management of various respiratory and hemodynamic complications seen in the ICU. These individuals are well-qualified to serve as general intensivists, either by attending to general critical care patients in other units, or by taking overflow patients into the NCCU. Reactivating retired physicians and APPs and carefully integrating them into the hospital provider pool may allow for additional resources in pandemic and disaster scenarios. The recruitment of these individuals and their deployment may depend upon the demandsupply relationship for a given hospital or region. Careful consideration of institutional standards, state licensing approvals, and governmental policies should occur prior to reactivation of staff members.

It is equally important to ensure that adequate neurocritical care expertise remains available to address the needs of patients with primary neurological emergencies, as well as those of critical care patients with secondary neurological complications. Neurointensivists and neurocritical care APPs can serve as a critical resource by providing consultative services, either in person or remotely. Similar to the SCCM model, a neurocritical care clinician may provide supervision, education, and recommendations to general intensivists, general neurologists, and providers from other backgrounds who may be called upon to care for complex patients with neurological diseases. To achieve these goals, effective use of telemedicine in patient care (both remotely as well as locally), and education should be considered. Depending on local resource availability, this may consist of secure applications with synchronous audio and visual support that are already embedded within an institutional electronic medical record versus utilization of external vendors or modalities. Maintaining flexible staffing models is crucial, as it is unlikely that any single solution will be universally applicable, and the needs of a particular institution will fluctuate over time. Close coordination between critical care clinicians, hospital administrators, and the local neurocritical care community will be required.

In such an environment, the need to preserve clinician well-being remains a priority. According to the Centers for Disease Control, at least 9282 health care workers in the US had contracted COVID-19 as of April 9th, 2020; this figure likely represents a significant underestimate due to insufficient testing [32] and general lack of screening at the time of this publication. More than half of these professionals (55\%) reported no known exposure outside of health care settings. In addition to the need to prioritize adequate access to PPE for staff, flexibility in
ICU workflow and workforce utilization should be evaluated to minimize the risk to personnel. Block scheduling, in which clinical assignments for individual providers are concentrated with designated periods of time away from the hospital, is an option to minimize exposure and transmission through asymptomatic spread as providers essentially self-isolate between clinical assignments. Clinical personnel should be encouraged to remain off campus when they are not engaged in direct patient care, and administrative and educational conferences should be held remotely. During clinical assignments, transitioning from bedside rounding to so-called table rounding is a reasonable strategy: by conducting clinical discussions in a conference room while adhering to physical distance recommendations or via conference calls away from patient care areas, the clinical team decreases the risk of prolonged exposure to contaminated surfaces, aerosolized particles, and asymptomatic team members. Unfortunately, this may also limit the ability of bedside nursing staff, respiratory therapists, pharmacists and other providers to participate in rounds. This drawback can be mitigated by the use of telephone or videoconferencing solutions. Telemedicine technology can also be used to facilitate patient examinations in the interest of minimizing movement into and out of patient rooms and team member exposure. Telemedicine has been widely studied in acute stroke care and shown to be beneficial [33-35]. Although there are less data on the use of telemedicine in neurocritical care specifically, there is evidence that robotic telepresence allows for rapid faceto-face attending physician response to ICU patients and results in decreased ICU cost and length of stay [36], as well as data supporting increased nursing satisfaction with implementation of robotic telepresence [37]. Telemedicine-based critical care services have expanded considerably in recent years $[38,39]$. One meta-analysis of telemedicine in critically ill patients demonstrated that telemedicine, compared with standard care, was associated with lower ICU mortality [risk ratio (RR) 0.79; 95\% confidence interval (CI), 0.65 to 0.96 ; nine studies, $n=23,526 ; I 2=70 \%$ ] and hospital mortality [40]. Incorporating this technology into the ICU provides an opportunity to limit exposure while simultaneously allowing sharing of expertise more broadly.

\section{Nursing, Pharmacy and Additional Healthcare Provider Staffing Considerations in Neurocritical Care}

Preparing hospital staff to meet increasing demands is essential and provides unique opportunities for systems to creatively meet health care needs. Hospital systems may attempt to increase staffing, reallocate staff, and design novel workflow models to ensure patient and 
staff safety. In the setting of redistribution of neurocritical care patients and/or providers, healthcare systems should maintain dedication to experienced neurocritical care providers caring for patients with acute neurological illness. These staff include but are not limited to dietitians, healthcare chaplains, nurses, pharmacists, physical and occupational therapists, respiratory therapists, and speech and language pathologists.

System strain may limit such staffing dynamics. In these cases, it may be useful to cross-train staff members from other specialty departments familiar with the neurological population to meet patient needs. Candidate populations include staff from other acute inpatient units, float pools, operating rooms, and stepdown units to care for critically ill patients under the direction and supervision of experienced NCCU staff. Nurses, pharmacists, and other staff with previous NCCU experience or retired healthcare providers may also be considered, depending on institutional and governmental regulations and policies. Training should ideally occur preemptively, rather than reactively, and should adjust to meet surge capacities.

As system strain increases, neurocritical care team members may be called upon to care for critically ill patients without acute neurological injuries. Neurocritical care practitioners are trained and prepared to meet such needs. Neurocritical care nurses are familiar with hemodynamic monitoring, mechanical ventilation, and vasopressor administration and have the foundation to care for critically ill patients in a variety of settings. When reallocating staff to an unfamiliar environment, offering support and necessary disease state education is key. Hybrid teams comprised of neurocritical care nurses, pharmacists, and APPs blended with those from other specialties may permit leveraging NCC expertise with surge deployment needs. Concerted efforts should be made to limit staff exposure to infected patients and minimize staff reallocation to and from infected and noninfected patient care areas.

When managing critically ill patients, finding ways to provide safe care at the bedside is of utmost concern. Attempts should be made to adhere to safe patientto-caregiver ratios. Optimization of existing technology within each hospital system should occur to ensure adequate patient monitoring. This may include the use of bedside video monitoring to allow the nurse to assist providers with examinations. Staff using advanced technology (multimodal monitoring, cardiac output monitoring, pupillometry, etc.) should be trained on proper use to prevent disease exposure and patient harm. The frequency of neurological examinations should be carefully considered for each patient, taking into account the patient's neurological injury, risk for neurological decline, time since symptom onset, medical comorbidities, and risk to the health care team caring for a patient with contagious illness. Bundling care along with medication administration ensures patient care needs are met, and unintentional harm is reduced. To reduce the risk of exposure to contagious illness, multiple visits to a patient's room may also be reduced by relocating frequently accessed equipment immediately outside the room using intravenous extension tubing, remote ventilator controls, and robotic telepresence.

\section{Pharmacy Considerations in Neurocritical Care}

As providers continue to aggressively work to manage critically ill patients, collaborating with pharmacy colleagues is crucial to allocate vital drug therapy, optimize drug treatment strategies, optimize medication administration, manage medication shortages, and mitigate the risk of medication errors, adverse effects and interactions. Patients who are severely affected during resource crises may require opioid analgesics, sedatives, and neuromuscular blockade. Supplies of commonly used drugs such as fentanyl, propofol, benzodiazepines, barbiturates, ketamine, and paralytics may be severely affected by drug shortages which may limit their availability for the management of acute pain, delirium, status epilepticus, targeted temperature management, and elevated intracranial pressures. Pharmacists can provide expert guidance to minimize the impact of medication shortages such as determining appropriate extension of expiration times, compounding medications on site, and changing medication routes as appropriate. Changes in medication administration may also be necessary to meet patient needs. In the case of airborne versus bloodborne pathogens, decreasing aerosolized medications may improve safety of administration. When possible, efforts should be made to utilize enteral dosing as a sparing strategy which may be especially beneficial in reducing intravenous (IV) medications on shortage. Likewise, in patients with contagious illness medication administration should be bundled and frequency adjusted appropriately to reduce exposure to bedside staff. When administering medications through extended IV tubing, drug-drug compatibility in the extended tubing should be evaluated by the pharmacist to ensure patient safety. This strategy also has the added benefit of conservation of PPE for the hospital through limitation of repeated room entries.

As with other health care practitioners, ensuring safety of critical care clinical pharmacists and pharmacy personnel is crucial in optimizing critical care delivery. Limiting pharmacist exposure to contagious patients and planning for early, pre-surge critical care cross-training among teams to assist with patient demands is key. In order to maintain physical distancing and as a result of 
resource limitations during pandemic medicine, dedicated pharmacists may not be available for neurocritical care rounds. Telepharmacy consultations may offer a solution to continued high-quality care and allow for pharmacists to teleround with the care teams.

\section{Education and Training}

The current global pandemic has significantly impacted health care delivery and therefore impacted education of medical, nursing, pharmacy, and other health professions students and trainees. Concerns for student and trainee safety and the need to preserve PPE render traditional methods of medical education impractical. Whenever possible, clinical experiences should be modified to minimize risk of exposure and maximize learning. While clinical rotations for medical, nursing, and pharmacy students have been suspended in many academic centers, residents and fellows remain an integral part of the healthcare workforce with a valuable skill set. It is imperative that residents and fellows be provided with a safe working environment, including adequate PPE and appropriate training in its use. In-person or online training in safety protocols should be made available prior to on-site clinical rotations. Hospital isolation guidelines and testing protocols should be readily accessible for reference. Implementation of dedicated procedure teams of experienced personnel can also reduce the number and time students and trainees are exposed to high-risk situations.

The clinical curriculum has the potential to be disrupted for almost every healthcare specialty. Cancelation of elective admissions and outpatient visits can prevent students and trainees from completing rotations; meanwhile, the increased volume of critical care patients may require that these individuals, regardless of prior experience, are redeployed to ICUs. As many neurology and neurosurgery residencies require an NCCU rotation, this provides an opportunity to resident trainees to develop a strong foundation in basic critical care principles. However, there is still an acute need for critical care education targeting a more general audience. In this respect, professional organizations can augment education to help bridge knowledge and experience gaps.

If these healthcare providers are unable to continue with their usual clinical responsibilities, alternate approaches such should be considered. Videoconferencing technology facilitates ongoing didactic conferences, and can also allow students and residents to participate in teaching rounds remotely. In recognition of increased use of telemedicine, the Accreditation Council for Graduate Medical Educations has accelerated the implementation of Common Program Requirements pertinent to telehealth visits [41]. Telemedicine platforms can permit trainees to participate in patient care while away from the hospital, or can be used to minimize contact with patients during on-site rotations.

Collaboration across disciplines and levels of training is necessary for adaptation to resource crises. Institutions may find innovative solutions to address limited PPE and global patient care processes through nontraditional channels. "Open-platform" venues allow for crowd-sourcing and real-time refinement of protocols and processes. Combining these efforts with data sharing through patient registries will enable quality improvement efforts, providing observational data to better inform patient care while awaiting the results of controlled trials. Supporting the continued development of regional and national platforms for development and dissemination of best practices benefits patient care and clinical education and can also facilitate integration of evidence-based medicine as research is published.

\section{Emotional and Psychiatric Support for Providers}

The phenomenon of burnout in critical care providers is well recognized. Ongoing exposure to trauma, tragedy, and death are catalysts unique to this area of medicine and these exposures are more intense during a pandemic. Contributing factors include longer working hours with limited resources in settings where their own existing support systems are unavailable and fear over possible self-infection or impacts to their families. Efforts to mitigate the consequences of burnout and promote resiliency are instrumental in preserving the well-being and mental health of the critical care workforce [42]. As such, providers should be supported in their roles through reduction of clerical burdens as much as is feasible, adherence to clear reporting structures to promote accountability and problem-solving, encouragement of open communication, and resources to pursue support for day-to-day responsibilities outside of the clinical environment. Mental health and well-being support along with access to counseling should be prioritized during the pandemic, and continued after acute patient care issues have returned to prepandemic levels of functioning. 


\section{Summary of key suggestions for neurocritical care resource utilization in pandemics}

Key suggestions

Systems of care

Emphasize continued appropriate triage of neurocritical care patients

- Transfer to higher level of care when required

- Utilization of telemedicine when available with patient retention at presenting hospital

Develop screening and testing pathways for those with contagious illness and cohort early and accordingly

Educate the public on infection control processes, and the need to seek care when required

\section{Inpatient care}

Prioritize care of neurocritically ill patients by experienced teams

Facilitate communications with patients' families

Avoid premature limitations on care

Neurocritical care unit staffing

Neurocritical care experts can be leveraged to provide intensive care to patients without primary neurologic illness

Implement interventions to prevent infections of health care workers:

- Minimize room entry

- Block scheduling

- Use of telemedicine to minimize physical contact

Cross-train nurses, pharmacists, and other providers early to extend expertise

\section{Pharmacy considerations}

Bundle medications and care interventions

Place IV pumps outside patients' rooms

Strategize to mitigate medication shortages:

Early transition to enteral route

Judicious extension of expiration times

Education and training

Utilize teleconferencing to minimize time spent at the hospital

Promote "open-platform" venues to facilitate collaboration while recognizing limitations

Facilitate disseminate of evidence-based practices

\section{Conclusions}

The occurrence of a pandemic results in a constantly changing healthcare delivery environment, which requires continual adaptation of rigid systems that are often designed to standardize care. Innovative models of neurocritical care delivery-from triage to dischargeare required to ensure patients continue to receive highquality, high-value neurocritical care in these challenging circumstances. Interprofessional neurocritical care providers have unique skills which can be leveraged to provide care to patients with both acute neurological and other diseases during a pandemic.

\footnotetext{
Author details

${ }^{1}$ Neurocritical Care, OhioHealth Riverside Methodist Hospital, 3535 Olentangy River Road, Columbus, OH 43214, USA. ${ }^{2}$ Departments of Neurology and Anesthesiology, University of Alabama at Birmingham, Birmingham, AL, USA. ${ }^{3}$ Department of Neurosurgery, Duke Health, Durham, NC, USA. ${ }^{4}$ Faculty of Medicine, Health Sciences Center, Kuwait University, Kuwait City, Kuwait. ${ }^{5}$ Departments of Pharmacotherapy and Outcomes Sciences and Neurosurgery, Virginia Commonwealth University, Richmond, VA, USA. ${ }^{6}$ Department of Neurology, NYU School of Medicine, New York, NY, USA. ${ }^{7}$ Departments of Neurology and Surgery, University of Massachusetts, Worcester, MA, USA.
}

${ }^{8}$ Department of Neurological Sciences, Rush University Medical Center, Chicago, IL, USA. ${ }^{9}$ Departments of Neurology, Neurosurgery, Anesthesia and Critical Care, University of Pennsylvania, Philadelphia, PA, USA. ${ }^{10}$ Department of Neurosurgery, McGovern Medical School, University of Texas Health Sciences Center, Houston, TX, USA. ${ }^{11}$ Department of Anesthesiology and Pain Medicine, Neurological Surgery, Harborview Medical Center, University of Washington, Seattle, WA, USA. ${ }^{12}$ Department of Neurology, The Ohio State University Wexner Medical Center, Columbus, OH, USA. ${ }^{13}$ Department of Pharmacy, The Ohio State University Wexner Medical Center, Columbus, $\mathrm{OH}$, USA. ${ }^{14}$ Departments of Neurology and Neurosurgery, New York Medical College, Valhalla, NY, USA. ${ }^{15}$ Interdepartmental Division of Critical Care Medicine, University of Toronto, Toronto, ON, Canada. ${ }^{16}$ Universidade Federal de São Paulo-UNIFESP and Hospital Israelita Albert Einstein, São Paulo, SP, Brazil. ${ }^{17}$ Department of Critical Care Medicine and Neurology, University of Pittsburgh, Pittsburgh, PA, USA. ${ }^{18}$ University of Southern California, Los Angeles, CA, USA. ${ }^{19}$ Department of Pharmacy Practice, College of Pharmacy, University of Illinois Hospital and Health Sciences System, Chicago, IL, USA. ${ }^{20}$ Department of Neurology, University of Pennsylvania, Philadelphia, PA, USA.

\section{Acknowledgements}

The authors would like to express deep appreciation for the support of the NCS Executive Committee, Ms. Sharon Allen, and Ms. Tissy Greene. Additionally we are grateful to Dr. Herb Fried, Dr. Lori Kennedy Madden, and the NCS Guidelines Committee.

\section{Author Contributions}

All authors contributed to the conception, drafting, and critical revision of the manuscript.

\section{Source of Support}

No grant support was received from any funding agency in the public, commercial, or not-for-profit sectors.

\section{Conflict of interest}

The authors declare that they have no conflict of interest.

\section{Ethical approval/Informed consent}

This manuscript does not consist of human subject research and therefore is not under the jurisdiction of an Institutional Review Board.

\section{Publisher's Note}

Springer Nature remains neutral with regard to jurisdictional claims in published maps and institutional affiliations.

Published online: 28 May 2020

\section{References}

1. World Health Organization. Coronavirus disease 2019 (COVID-19) situation report-94. Geneva: World Health Organization; 2020. p. 12.

2. Ji Y, Ma Z, Peppelenbosch MP, Pan Q. Potential association between COVID-19 mortality and health-care resource availability. Lancet Glob Health. 2020;8(4):e480.

3. Moghadas SM, Shoukat A, Fitzpatrick MC, et al. Projecting hospital utilization during the COVID-19 outbreaks in the United States. Proc Natl Acad Sci USA. 2020;117(16):9122-6.

4. Emanuel EJ, Persad G, Upshur R, et al. Fair allocation of scarce medical resources in the time of Covid-19. N Engl J Med. 2020.

5. Diringer MN, Edwards DF. Admission to a neurologic/neurosurgical intensive care unit is associated with reduced mortality rate after intracerebral hemorrhage. Crit Care Med. 2001;29(3):635-40.

6. Varelas PN, Schultz L, Conti M, et al. The impact of a neuro-intensivist on patients with stroke admitted to a neurosciences intensive care unit. Neurocrit Care. 2008;9(3):293-9.

7. Suarez II, Zaidat OO, Suri MF, et al. Length of stay and mortality in neurocritically ill patients: impact of a specialized neurocritical care team. Crit Care Med. 2004:32(11):2311-7. 
8. Bershad EM, Feen ES, Hernandez OH, Suri MF, Suarez JI. Impact of a specialized neurointensive care team on outcomes of critically ill acute ischemic stroke patients. Neurocrit Care. 2008;9(3):287-92.

9. Varelas PN, Conti MM, Spanaki MV, et al. The impact of a neurointensivistled team on a semiclosed neurosciences intensive care unit. Crit Care Med. 2004;32(11):2191-8.

10. Varelas PN, Eastwood D, Yun HJ, et al. Impact of a neurointensivist on outcomes in patients with head trauma treated in a neurosciences intensive care unit. J Neurosurg. 2006;104(5):713-9.

11. Mirski MA, Chang CW, Cowan R. Impact of a neuroscience intensive care unit on neurosurgical patient outcomes and cost of care: evidence-based support for an intensivist-directed specialty ICU model of care. J Neurosurg Anesthesiol. 2001;13(2):83-92.

12. Samuels O, Webb A, Culler S, Martin K, Barrow D. Impact of a dedicated neurocritical care team in treating patients with aneurysmal subarachnoid hemorrhage. Neurocrit Care. 2011;14(3):334-40.

13. Josephson SA, Douglas VC, Lawton MT, et al. Improvement in intensive care unit outcomes in patients with subarachnoid hemorrhage after initiation of neurointensivist co-management. J Neurosurg. 2010:112(3):626-30

14. Knopf L, Staff I, Gomes J, McCullough L. Impact of a neurointensivist on outcomes in critically ill stroke patients. Neurocrit Care. 2012;16(1):63-71.

15. Burns JD, Green DM, Lau H, et al. The effect of a neurocritical care service without a dedicated neuro-ICU on quality of care in intracerebral hemorrhage. Neurocrit Care. 2013;18(3):305-12.

16. Suarez J, Martin RH, Bauza C, et al. worldwide organization of neurocritical care: results from the PRINCE study part 1. Neurocrit Care. 2020;32(1):172-9.

17. Venkatasubba Rao CP, Suarez Jl, Martin RH, et al. Global survey of outcomes of neurocritical care patients: analysis of the PRINCE study part 2. Neurocrit Care. 2020;32(1):88-103.

18. Jeong JH, Bang J, Jeong W, et al. A dedicated neurological intensive care unit offers improved outcomes for patients with brain and spine injuries. J Intensive Care Med. 2019;34(2):104-8.

19. Soliman I, Aletreby WT, Faqihi F, et al. Improved outcomes following the establishment of a neurocritical care unit in Saudi Arabia. Crit Care Res Pract. 2018;2018:2764907

20. Egawa S, Hifumi T, Kawakita K, et al. Impact of neurointensivist-managed intensive care unit implementation on patient outcomes after aneurysmal subarachnoid hemorrhage. J Crit Care. 2016;32:52-5.

21. Harrison DA, Prabhu G, Grieve R, et al. Risk adjustment in neurocritical care (RAIN) - prospective validation of risk prediction models for adult patients with acute traumatic brain injury to use to evaluate the optimum location and comparative costs of neurocritical care: a cohort study. Health Technol Assess. 2013;17(23):1-350.

22. Kramer AH, Zygun DA. Neurocritical care: why does it make a difference? Curr Opin Crit Care. 2014;20(2):174-81.

23. McCredie VA, Alali AS, Scales DC, et al. Impact of ICU structure and processes of care on outcomes after severe traumatic brain injury: a multicenter cohort study. Crit Care Med. 2018;46(7):1139-49.

24. Moheet AM, Livesay SL, Abdelhak T, et al. Standards for neurologic critical care units: a statement for healthcare professionals from the neurocritical care society. Neurocrit Care. 2018;29(2):145-60.
25. Kaplan L, Moheet AM, Livesay SL, et al. A perspective from the neurocritical care society and the society of critical care medicine: team-based care for neurological critical illness. Neurocrit Care. 2020;32(2):369-72.

26. Christian MD, Sprung CL, King MA, et al. Triage: care of the critically ill and injured during pandemics and disasters: CHEST consensus statement. Chest. 2014;146(4 Suppl):e61S-74S.

27. Oxley TJ, Mocco J, Majidi S, et al. Large-vessel stroke as a presenting feature of Covid-19 in the young. N Engl J Med. 2020;382:e60.

28. Khan MW, Muehlschlegel S. Shared decision making in neurocritical care. Neurosurg Clin N Am. 2018;29(2):315-21.

29. Halpern NA, Tan KS, DeWitt M, Pastores SM. Intensivists in US Acute Care Hospitals. Crit Care Med. 2019:47(4):517-25.

30. Kleinpell RM, Grabenkort WR, Kapu AN, Constantine R, Sicoutris C. Nurse practitioners and physician assistants in acute and critical care: a concise review of the literature and data 2008-2018. Crit Care Med. 2019:47(10):1442-9.

31. Halpern NA, Tan KS, SCCM Ventilator Taskforce. US ICU resource availability for COVID-19. Los Angeles: Society of Critical Care Medicine; 2020.

32. Report, M.M.W. Characteristics of health care personnel with COVID-19United States, February 12-April 9, 2020, MMWR, Editor 2020. p. 477-81.

33. Schwamm LH, Holloway RG, Amarenco $P$, et al. A review of the evidence for the use of telemedicine within stroke systems of care: a scientific statement from the American Heart Association/American Stroke Association. Stroke. 2009;40(7):2616-34.

34. Hatcher-Martin JM, Adams JL, Anderson ER, et al. Telemedicine in neurology: telemedicine work group of the American Academy of Neurology update. Neurology. 2020;94(1):30-8

35. Wechsler LR, Tsao JW, Levine SR, et al. Teleneurology applications: report of the telemedicine work group of the American Academy of Neurology. Neurology. 2013;80(7):670-6.

36. Vespa PM, Miller C, Hu X, et al. Intensive care unit robotic telepresence facilitates rapid physician response to unstable patients and decreased cost in neurointensive care. Surg Neurol. 2007:67(4):331-7.

37. Rincon F, Vibbert $M$, Childs V, et al. Implementation of a model of robotic tele-presence (RTP) in the neuro-ICU: effect on critical care nursing team satisfaction. Neurocrit Care 2012:17(1):97-101.

38. Subramanian S, Pamplin JC, Hravnak M, et al. Tele-critical care: an update from the society of critical care medicine Tele-ICU Committee. Crit Care Med. 2020;48(4):553-61

39. Kahn JM, Cicero BD, Wallace DJ, Iwashyna TJ. Adoption of ICU telemedicine in the United States. Crit Care Med. 2014;42(2):362-8.

40. Wilcox ME, Adhikari NK. The effect of telemedicine in critically ill patients: systematic review and meta-analysis. Crit Care. 2012;16(4):R127.

41. Nasca T. ACGME response to the Coronavirus (COVID-19).

42. Kleinpell R, Moss M, Good VS, Gozal D, Sessler CN. The critical nature of addressing Burnout prevention: results from the critical care societies $\mathrm{Col}$ laborative's national summit and survey on prevention and management of Burnout in the ICU. Crit Care Med. 2020;48(2):249-53. 